\title{
Incidence of carnitine deficiency in patients with cancer pain: A pilot study
}

\author{
KIYOHIRO SAKAI $^{1}$, HIROMICHI MATSUOKA $^{1,2}$, YOICHI OHTAKE $^{1}$, CHIHIRO MAKIMURA $^{1}$, \\ HIROAKI IZUMI $^{1}$, YOSHIHIKO FUJITA ${ }^{3}$, MASATOMO OTSUKA ${ }^{4}$, JUNJI TSURUTANI ${ }^{5}$, \\ KAZUTO NISHIO $^{3}$, KAZUHIKO NAKAGAWA ${ }^{5}$ and ATSUKO KOYAMA ${ }^{1}$ \\ Departments of ${ }^{1}$ Psychosomatic Medicine, ${ }^{2}$ Palliative Care Center, and ${ }^{3}$ Genome Biology, \\ Kindai University Faculty of Medicine, Osaka 589-8511; ${ }^{4}$ Department of Palliative Care, Sakai Hospital, \\ Kindai University Faculty of Medicine, Osaka 590-0132; 5 Department of Medical Oncology, \\ Kindai University Faculty of Medicine, Osaka 589-8511, Japan
}

Received June 22, 2016; Accepted August 18, 2016

DOI: $10.3892 /$ mco.2017.1159

\begin{abstract}
Carnitine deficiency is reportedly associated with increased pain sensation in diabetes mellitus and fibromyalgia, but the association between serum carnitine concentration and cancer pain has not been fully elucidated. We investigated the incidence of carnitine deficiency in patients with cancer pain, and examined the effect of the patients' demographic and clinical characteristics on pain intensity and carnitine deficiency. The serum carnitine concentration was measured in 50 patients with cancer pain receiving non-steroidal anti-inflammatory drugs, but not opioids. Multivariate regression analysis was used to determine the association of carnitine concentration, pain intensity, age and gender with hemoglobin and C-reactive protein (CRP) concentrations. Carnitine deficiency was detected in 9 of the patients $(18.0 \%)$ and found to be significantly correlated with an elevated CRP concentration $(\mathrm{P}=0.039)$. In conclusion, although there does not appear to be an association between carnitine deficiency and cancer pain, it may be affected by inflammation or infection.
\end{abstract}

\section{Introduction}

One-third of patients treated for cancer report pain; among those with advanced disease, more than two-thirds report nociceptive and/or neuropathic pain that may occur continuously or as breakthrough pain $(1,2)$. Carnitine is a low-molecular weight amino acid derivative that plays a role in cellular metabolism and transportation of fatty acids into the mitochondria (3).

Correspondence to: Dr Hiromichi Matsuoka, Department of Psychosomatic Medicine, Kindai University Faculty of Medicine, 377-2 Ohno-higashi, Osaka-Sayama, Osaka 589-8511, Japan

E-mail: matsuoka_h@med.kindai.ac.jp

Key words: cancer, carnitine, C-reactive protein, pain
Primary carnitine deficiency is a genetic defect involving the active transport across the plasmalemmal membrane (4). Secondary carnitine deficiencies are associated with another disorder, such as liver or kidney disease, a defect in fatty acid metabolism, or certain pharmacological agents (5-7).

Depletion of serum carnitine may also exert profound effects on cardiac and skeletal muscle, serum lipid concentrations and stability of red blood cells $(8,9)$. Carnitine deficiency may also affect pain transmission: Deficiency is associated with increased pain intensity in diabetes mellitus, HIV infection and fibromyalgia, which may be addressed by carnitine supplementation (10-12). Several mechanisms have been proposed to explain how carnitine may mediate analgesia, including promotion of nerve conduction $(13,14)$, anti-depressant activity (12) and improved blood flow to skeletal muscle (12). To the best of our knowledge, the association between serum carnitine concentration and cancer pain has not been investigated to date.

\section{Patients and methods}

Patient inclusion criteria. Patients with cancer pain who presented to the Kindai University Faculty of Medicine or the Sakai Hospital (Kindai University Faculty of Medicine, Osaka, Japan) between July 1, 2011 and December 31, 2012, were screened.

The inclusion criteria were as follows: Cancer pain; an analgesic regime at enrollment of a licensed dose of a non-steroidal anti-inflammatory drug; and no current or prior treatment with an opioid. Patients taking opioids and those who were considered by the investigators not to be suitable for participation were excluded. The study protocol was approved by the Ethics Committee of Kindai University of Medicine, Japan. Written informed consent to participate was obtained from all patients.

Patient demographic and clinical characteristics. Recorded patient information included age, gender, physical status as determined by the Eastern Cooperative Oncology Group performance status (PS), pain intensity (numerical rating scale 
Table I. Demographic and clinical characteristics of the patients $(n=50)$.

\begin{tabular}{lc}
\hline Characteristics & Number of patients \\
\hline Age, years & \\
$<65$ & 36 \\
$\geq 65$ & \\
Gender & 25 \\
Men & 25 \\
Women & \\
Performance status & 34 \\
0-2 & 16 \\
$3-4$ & \\
Tumor types & 21 \\
Lung & 8 \\
Colorectal & 5 \\
Gastric & 3 \\
Breast & 2 \\
Gallbladder & 2 \\
Pancreatic & 9 \\
Others & 2 \\
\hline
\end{tabular}

of pain), tumor types, presence of metastatic disease, pleural effusion, ascites, constipation, nausea or sedation, blood concentration of hemoglobin, white blood cell and platelet counts, and serum concentrations of protein, albumin, creatinine and $\mathrm{C}$-reactive protein (CRP).

Endpoint. The primary aim of this study was to establish the incidence of carnitine deficiency in patients with cancer pain. The secondary aim was to investigate whether there were associations between carnitine deficiency and patient demographic and clinical characteristics.

Measurement of serum carnitine concentration. Venous blood samples were collected in a 5-ml EDTA-sodium blood tubes prior to the initiation of opioid treatment, and separated sera were stored at $-80^{\circ} \mathrm{C}$ until required for assays. For carnitine assays, the sample was centrifuged for $15 \mathrm{~min}$ at 3,000 $\mathrm{x} g$, then 500- $\mu 1$ aliquots of plasma were removed and stored at $-80^{\circ} \mathrm{C}$ until required for assays. The enzymatic cycling method was used (15). We measured free carnitine and acylcarnitine concentrations and their sum was reported as the total carnitine concentration. All carnitine assays were undertaken at the BML General Laboratory, Saitama, Japan.

Definition of carnitine deficiency. Carnitine deficiency was defined as an acylcarnitine to free carnitine ratio of $>0.4$, or a free carnitine concentration $<35 \mu \mathrm{mol} / 1$ in men or $<25 \mu \mathrm{mol} / 1$ in women.

Statistical analysis. A bivariate analysis was first undertaken, with carnitine deficiency as the dependent variable and patient clinical and demographic characteristics as independent variables. Independent variables with a P-value $\geq 0.2$ were excluded from further analyses, whereas variables with a P-value $<0.2$ were subjected to multiple logistic regression analysis. All statistical analyses were performed using SPSS software (version 19.0; SPSS Japan Inc., Tokyo).

\section{Results}

Patient characteristics. A total of 50 patients met the inclusion criteria and were included in the analysis. Their demographic and clinical characteristics are summarized in Table I.

The mean age \pm standard deviation of the cohort was $68.5 \pm 9.9$ years; 25 patients were men $(50.0 \%)$ and 25 were women $(50.0 \%) ; 34$ patients had a PS of $0-2(68.0 \%)$. The primary tumor was in the lung in 21 cases, colon or rectum in 8 , stomach in 5 , breast in 3 , gallbladder in 2 and pancreas in 2 cases; 9 patients had other cancers, including 4 in whom the primary was unknown. Carnitine deficiency was identified in 9 patients $(18.0 \%)$. Five variables were selected for multivariate analysis (age, gender, pain intensity, blood hemoglobin concentration and serum concentration of C-reactive protein). Multivariate analysis demonstrated that carnitine deficiency was not correlated with pain intensity $(\mathrm{P}=0.463)$, but was significantly correlated with elevated serum CRP concentration $(\mathrm{P}=0.039$, Table II).

\section{Discussion}

To the best of our knowledge, our study is the first to investigate the incidence of carnitine deficiency in patients with cancer pain. Our main finding was that the incidence of carnitine deficiency in a cohort of 50 patients with cancer pain was $18.0 \%$. Rabito et al (16) reported that the plasma concentration of free carnitine was significantly lower in cancer patients, $80 \%$ of whom had insufficient dietary protein intake and low body fat reserves; their cohort consisted of patients awaiting either palliative or curative surgery for esophageal or gastric cancer, who would have been at high risk of dietary insufficiency and malnutrition. Our cohort was significantly less likely to be carnitine-deficient. This may be explained by the presence of a majority $(68 \%)$ with a PS of 0,1 or 2 , indicating that only few patients were cachectic and dietary insufficiency was less likely.

We also found that carnitine deficiency was significantly associated with elevated serum CRP concentrations. In patients with head and neck cancer, the presence of pain is reportedly associated with elevated serum CRP concentrations (17). Our study included no participants with head and neck cancer. It may be helpful to examine the incidence of carnitine deficiency specifically in patients with head and neck cancer, in order to establish whether there is an association between serum carnitine and CRP concentrations. In patients with end-stage kidney disease undergoing maintenance hemodialysis, an elevated serum CRP concentration was improved by carnitine supplementation, suggesting that carnitine exerts an anti-inflammatory effect (18). Further investigation is required to establish whether cancer pain may also resolve with carnitine supplementation.

Our study had several important limitations. First, the study was conducted in a single center and the size of the 
Table II. Independent determinants of carnitine deficiency.

Partial regression coefficient $(95 \% \mathrm{CI})$

\begin{tabular}{llll} 
Item & Univariate model & Multivariate model & P-value \\
Age & $1.046(0.9601 .140)$ & $1.088(0.9791 .210)$ & 0.119 \\
Gender & $0.432(0.9501 .966)$ & $0.310(0.0511 .892)$ & 0.204 \\
Hemoglobin & $0.978(0.6671 .434)$ & $1.285(0.7752 .131)$ & 0.331 \\
C-reactive protein & $1.127(1.0011 .269)$ & $1.161(1.0081 .338)$ & 0.039 \\
Pain intensity & $1.076(0.7881 .470)$ & $1.150(0.7921 .669)$ & 0.463 \\
\hline
\end{tabular}

CI, confidence interval.

cohort was relatively small, so it is difficult to draw firm conclusions on the statistical (and clinical) significance of some of the associations we examined. Second, patients with possible drug-induced carnitine deficiency were not excluded, as we did not record the complete drug histories (including chemotherapy) of the participants.

In conclusion, the incidence of carnitine deficiency in a cohort of 50 patients with cancer pain was $18.0 \%$. Although carnitine deficiency was not associated with pain intensity, there appeared to be an association between carnitine deficiency and elevated serum CRP concentration.

\section{Acknowledgements}

This study was supported by the Third-Term Comprehensive 10-Year Strategy for Cancer Control and a Grant-in-Aid for Cancer Research from the Ministry of Health, Labour and Welfare of Japan (H22-037) and funds from Merck Sharp and Dohme (Tokyo, Japan). We would like to thank Mrs. Tomoko Kitayama, Mrs. Erina Hatashita, Mrs. Kiyoko Kuwata, Mrs. Haruka Yamaguchi, Mr. Hiromasa Wadaguri and Mrs. Akiko Mizumoto for their technical assistance. We would also also like to thank Dr Richard Simon and Dr Amy Peng for providing us with the BRB ArrayTools software.

\section{References}

1. World Health Organization: Cancer Pain Relief. 2nd edition. World Health Organization, Geneva, 1996.

2. Caraceni A and Portenoy RK: An international survey of cancer pain characteristics and syndromes. IASP Task Force on cancer Pain. International association for the study of pain. Pain 82: 263-274, 1999.

3. Indiveri C, Iacobazzi V, Tonazzi A, Giangregorio N, Infantino V, Convertini P, Console L and Palmieri F: The mitochondrial carnitine/acylcarnitine carrier: Function, structure and physiopathology. Mol Aspects Med 32: 223-233, 2011.

4. Scholte HR, Rodrigues Pereira R, de Jonge PC, Luyt-Houwen IE, Hedwig M, Verduin M and Ross JD: Primary carnitine deficiency. J Clin Chem Clin Biochem 28: 351-357, 1990.

5. Duran M, Loof NE, Ketting D and Dorland L: Secondary carnitine deficiency. J Clin Chem Clin Biochem 28: 359-363, 1990.
6. Famularo G, Matricardi F, Nucera E, Santini G and De Simone C: Carnitine deficiency: Primary and secondary syndromes. In: Carnitine Today. De Simone C, Famularo G (eds). R.G. Landes Company, Austin, TX, pp119-161, 1997.

7. Pons R and De Vivo DC: Primary and secondary carnitine deficiency syndromes. J Child Neurol 10 (Suppl 2): S8-S24, 1995.

8. Eknoyan G, Latos DL and Lindberg J; National Kidney Foundation Carnitine Consensus Conference: Practice recommendations for the use of L-carnitine in dialysis-related carnitine disorder. National kidney foundation carnitine consensus conference. Am J Kidney Dis 41: 868-876, 2003.

9. Bonomini M, Zammit V, Pusey CD, De Vecchi A and Arduini A: Pharmacological use of L-carnitine in uremic anemia: Has its full potential been exploited? Pharmacol Res 63: 157-164, 2011.

10. De Grandis D and Minardi C: Acetyl-L-carnitine (levacecarnine) in the treatment of diabetic neuropathy. A long-term, randomised, double-blind, placebo-controlled study. Drugs R D 3: 223-231, 2002.

11. Scarpini E, Sacilotto G, Baron P, Cusini M and Scarlato G: Effect of acetyl-L-carnitine in the treatment of painful peripheral neuropathies in HIV+ patients. J Peripher Nerv Syst 2: 250-252, 1997.

12. Rossini M, Di Munno O, Valentini G, Bianchi G, Biasi G, Cacace E, Malesci D, La Montagna G, Viapiana O and Adami S: Double-blind, multicenter trial comparing acetyl l-carnitine with placebo in the treatment of fibromyalgia patients. Clin Exp Rheumatol 25: 182-188, 2007.

13. Onofrj M, Ciccocioppo F, Varanese S, di Muzio A, Calvani M, Chiechio S, Osio M and Thomas A: Acetyl-L-carnitine: From a biological curiosity to a drug for the peripheral nervous system and beyond. Expert Rev Neurother 13: 925-936, 2013.

14. Sima AA: Acetyl-L-carnitine in diabetic polyneuropathy: Experimental and clinical data. CNS Drugs 21 (Suppl 1): S13-S23, 45-46, 2007.

15. Obón JM, Buendía B, Cánovas M and Iborra JL: Enzymatic cycling assay for D-carnitine determination. Anal Biochem 274: 34-39, 1999.

16. Rabito EI, Leme IA, Demenice R, Portari GV, Jordão AA Jr, dos Santos JS and Marchini JS: Lower carnitine plasma values from malnutrition cancer patients. J Gastrointest Cancer 44: 362-365, 2013.

17. Oliveira KG, von Zeidler SV, Lamas AZ, Podestá JR, Sena A, Souza ED, Lenzi J, Lemos EM, Gouvea SA and Bissoli NS: Relationship of inflammatory markers and pain in patients with head and neck cancer prior to anticancer therapy. Braz J Med Biol Res 47: 600-604, 2014.

18. Chen Y, Abbate M, Tang L, Cai G, Gong Z, Wei R, Zhou J and Chen X: L-Carnitine supplementation for adults with end-stage kidney disease requiring maintenance hemodialysis: A systematic review and meta-analysis. Am J Clin Nutr 99: 408-422, 2014. 